\title{
Ciencia, revolución y nacimiento de la sociología ${ }^{1}$ Science, revolution and birth of sociology
}

Recibido: 09 de febrero de 2016 - Revisado: 28 de junio de 2016 - Aceptado: 02 de diciembre de 2016.

\section{Francisco Joaquín Cortés García ${ }^{2}$}

\section{Resumen}

La École Polytechnique fue una institución académica, revolucionaria y científica fundada a finales del siglo XVIII en Francia. La escuela reunió a los principales científicos de la época y utilizó los principios revolucionarios para instaurar la profesión del ingeniero, del concepto actual de comunidad científica y de la moderna vinculación entre el saber y el aparato del Estado. En el seno de dicha institución educativa surgió la sociología como ciencia, bajo una concepción positivista. En este artículo analizamos los estrechos vínculos entre la creación de la École Polytechnique y su importancia en el nacimiento de la sociología.

\section{Palabras Clave}

Ingeniero, École Polytechnique, sociología, nacimiento de la sociología.

\begin{abstract}
The École Polytechnique was an academic, revolutionary and scientific institution founded at the end of the 18th century in France. The school brought together the leading scientists of the time and used revolutionary principles to establish the profession of engineer, the current concept of the scientific community and the modern link between knowledge and the state apparatus. Within the educational institution sociology emerged as a science, under a positivist conception. In this article we analyze the close links between the creation of the École Polytechnique and its importance in the birth of sociology.
\end{abstract}

\section{Keywords}

Engineer, École Polytechnique, sociology, birth of sociology.
1 Artículo de investigación de la Universidad Internacional de la Rioja (UNIR), España.

${ }^{2}$ Doctor en Economía, profesor de la Universidad Internacional de la Rioja (UNIR) (España), investigador de la Universidad Autónoma de Chile y profesor de la Universidad Cooperativa de Colombia. Pertenece al consejo editorial de numerosas revistas académicas.

Correo electrónico: franciscojoaquincortesgarcia@gmail.com

Para citar este artículo use: Cortés, F. (2017). Ciencia, revolución y nacimiento de la sociología. Civilizar Ciencias Sociales y Humanas, 17(33), 163-176. doi: $10.22518 / 16578953.906$ 


\section{Introducción}

\section{La École Polytechnique, sistema educativo instaurado por Napoleón}

El emperador francés Napoleón Bonaparte concibió el sistema educativo como uno de los cimientos fundamentales para asentar la nueva sociedad francesa, que empezaba a vertebrarse tras la agitación y los desórdenes revolucionarios. Considerando que el sistema educativo heredado del Ancien Régime (Antiguo Régimen) y de la gran Revolución política debería ponerse al servicio del Estado, apostó por la laicidad, la uniformidad y la centralidad educativa.

La estructura principal del sistema de enseñanza napoleónico se apoya en tres pilares capitales: la Universidad, entendida como conjunto de centros y establecimientos de enseñanza superior; los Liceos, que son los centros de enseñanza secundaria, cuyos alumnos eran sometidos a un régimen disciplinario prácticamente de carácter "espartano"; y las Facultades, encargadas de la formación y promoción de profesores, docentes, juristas y médicos. Toda esta estructura anudada de forma arborescente y centralizada en un Ministerio de París.

Además, se constituyen las escuelas superiores especiales o "grandes escuelas" como centros docentes e investigadores externos a la Universidad: de ahí la École Poytechnique, donde casi todos sus miembros se dedicaban de forma simultánea tanto a la docencia como a la investigación, teniendo los investigadores una gran autonomía en las determinaciones, objetivos y resultados de su investigación. En concreto, este modelo de escuela superior sirvió, de forma inequívoca, de ejemplo para instituciones politécnicas de otros países como Alemania, Austria y Estados Unidos, y cuyo legado, en muchos aspectos, se puede percibir en nuestros días. De hecho, la École Polytechnique no fue una institución de enseñanza más, sino un hito fundamental en la historia de la ciencia, en la historia y la práctica de la ingeniería $\mathrm{y}$, obviamente, en la historia de los sistemas e instituciones educativos y docentes. A diferencia de Thomas de la Marne, que proponía suprimirla, Cabanis la defendió poniendo de manifiesto la naturaleza y la importancia de este centro docente en el ámbito del sistema escolar y en el conjunto de la República: "Les matières d'enseignement, ses professeurs illustres, les élèves distingués qu'elle a déjà fournis aux divers services publics, suffiraient à la défendre" (Picavet, 1975, p. 219). Por su parte, Picavet (1975) señala que «L'École Polytechnique, organisée en l'an III, eut pour professeurs les savants les plus illustres, Lagrange, Prony, Monge, Berthollet, Fourcroy, Chaptal, Vaquelin et Guyton de Morveau. Elle devait former des ingénieurs civils et militaires, enseigner les principes généraux des sciences" ${ }^{\prime 2}$ (p. 67).

Más aún, definitivamente es un ejemplo de la fusión, fértil alianza y profunda sinergia entre el poder, el mecenazgo, la incipiente industria, los intereses de los banqueros, los industriales y los burgueses y la investigación y la docencia. Las palabras del conde de Saint-Simon, "el gran espíritu politécnico y positivo", son un hecho demostrativo de esta síntesis/alianza, inédita en la historia: "construye una tupida red de relaciones sociales con científicos de diferentes disciplinas, empresarios y banqueros" (Coller, 2003, p.55).

La euforia que encarna el espíritu científico es una realidad en el siglo XVIII, una ciencia que redescubre el atomismo, logra notorios avances en el campo de la astronomía, de la química, de la biología, de la taxonomía en su sentido más extenso, del conocimiento de la materia, etcétera. Esta nueva emulsión de la ciencia se traslada, de una forma $u$ otra, a los países más avanzados desde el punto de vista económico, cultural, democrático y social bajo distintas concepciones o tendencias ideológicas: revolucionarias unas, reformistas otras o conservadoras algunas. En Inglaterra, se crean sociedades científicas, se fomentan las escuelas privadas; en Alemania, se opta por el reformismo en la enseñanza y se crea la Universidad de 
Berlín en 1810; pero es en Francia donde se evidencia con más fuerza lo más avanzado, si cabe, de esta euforia científica, positivista, fáustica ${ }^{3}$ y tecnológica, cuya expresión más elocuente, celebérrima y representativa será la Escuela Politécnica, que asumirá el proyecto educativo napoleónico, solidario con la nueva sociedad industrial, "la nueva sociedad positiva", con la abolición de la vieja aristocracia ociosa y de gran parte de sus privilegios y prerrogativas, $\mathrm{y}$, por supuesto, propiciadora de una profunda transformación social, económica y política basada en criterios científicos, extremadamente racionales, naturales, positivos, empíricos y netamente secularizados.

La historia de la institución se ha abordado desde muchos puntos de vista, pero han sido autores como Fourcy (1828), Picon (1994) o Shinn (1980) los que le dieron el enfoque que se recoge en este trabajo y que destacó Hayek (2003): un enfoque asociado al nacimiento de la ciencia moderna y de la sociología como disciplina.

\section{Presentación de Resultados: La École Polytechnique y los orígenes de la sociología}

En el imaginario colectivo de los franceses, la École Polytechnique es una institución que aún está viva, después de más de dos siglos, y es considerada claro símbolo de la grandeza nacional. La École Centrale des Travaux Publics (Escuela central de trabajos públicos), que un año más tarde tomará el nombre definitivo, hasta nuestros días, de École Polytechnique, fue creada en 1794 encarnando los ideales del siglo de la Ilustración y de la Enciclopedia, siendo firmemente apoyada por el mecenazgo de Napoleón Bonaparte y tácitamente por la incipiente burguesía industrial. Es, asimismo, la primera de este tipo del grupo de escuelas que fueron estableciéndose en Europa durante el siglo XIX.

Indiscutiblemente, se constituyó en el pilar fundamental del sistema de las grandes écoles (grandes escuelas) de Francia y ponía de manifiesto el nuevo poder burgués procedente tanto de la Revolución política francesa como de la Revolución Industrial que se inició en Inglaterra. De hecho, bajo este patrón educativo y académico-organizacional se crearon establecimientos politécnicos en ciudades como Praga (1806), Viena (1815), Berlín (1821), Zúrich (1855), Delft (1864), entre otras. Incluso en Norteamérica tuvo una influencia decisiva en algunas instituciones y centros académicos de gran prestigio, tanto de carácter civil como de carácter militar. De hecho, la Academia Militar de Estados Unidos ubicada en West Point, fue creada bajo la inspiración, el ideario y los fundamentos docentes y organizativos de esta institución.

La École Polytechnique, creada por la Convención, venía a recoger el espíritu científico que emergió de la Ilustración francesa y que bajo la epopeya politécnica se transformaría mediante la concreción de dos nuevas manifestaciones del espíritu: el positivismo y el cientismo, que tendrán importantes repercusiones en el pensamiento y en el desarrollo de la ciencia hasta nuestros días. Decididamente, fue una creación que los tiempos y que la incipiente sociedad industrial exigían. De hecho, fue una institución que estaba tácitamente predicha e ideada con anterioridad, y que el propio Donald Cardwell asimila con el sistema métrico: dos "acontecimientos que podrían haberse previsto o estaban incluso gestándose antes de 1789" (Cardwell, 2001). Hallando sus raíces más inmediatas en las viejas escuelas de ingenieros del Ancien Régimen, esta privilegiada institución se basó en un saber tecnocrático, y sus miembros indudablemente tuvieron el orgullo de casta de ser los mayores servidores del Estado y del interés general, capaces de materializar la utopía educativa del siglo de la Luces y de la Revolución plasmada en los nuevos Catálogos declarativos, así como la Idea hegeliana en el desarrollo de la historia. A su vez, permitiría al demolido y caótico Estado francés, que salía de la gran Revolución política de fin de siglo, do- 
tarse de los cuadros de funcionarios, ingenieros (tanto civiles como militares) y tecnócratas que requería para vertebrar su nueva estructura burguesa de organización del poder, bajo la alianza del poder financiero, el poder industrial, el poder político y el poder ligado al conocimiento, a la ciencia y a los nuevos desarrollos de la tecnología.

La École Polytechnique se constituyó, sin lugar a dudas, en el hito histórico que de forma inequívoca fue el punto de partida de la estructura de las grandes escuelas superiores que aglutinarían la enseñanza científica y tecnológica, con un carácter netamente diferenciado de las universidades. La práctica de la ingeniería francesa y de sus instituciones académicas y formativas se difundiría por todo el continente debido al propio poder político y militar de la Francia del momento; un país que según la socióloga Johan Heilbron (1995), estaba a la cabeza de Europa en las principales ciencias y disciplinas del saber: "en las ciencias matemáticas, físicas, médicas, y muy posiblemente también en las ciencias químicas y naturales" (ÁlvarezUría \& Varela, 2004). Nos referimos a la época gloriosa en la que se data el nacimiento de la École Polytechnique, cuando se crean en Francia los grandes manuales de ingeniería tal como se conciben en la actualidad. Hablamos de obras tan importantes y aceptadas como La Nouvelle architecture hydraulique (la Nueva arquitectura hidráulica), de Riche de Prony (1790, 1796), reconocida incluso en Gran Bretaña como la obra que ofrecía la mejor exposición de la máquina de vapor, o el Essai sur la composition de machines (Ensayo sobre la composición de las máquinas) de Lanz y Betancourt (1808), que seguía los trabajos de Lazare Carnot y Monge (Cardwell, 2001). A estos manuales, y siguiendo con la enumeración que Donald Cardwell (2001) realiza en su obra Historia de la tecnología, habría que añadir el Traité élémentaire des Machines (Tratado elemental de las máquinas) de Hachette (1811); el Essai sur la science des machines (Ensayo sobre la ciencia de las máquinas) de Guenyveau (1810); la versión revi- sada de la Architecture hydraulique de Belidor, por Navier (1819); De la richesse minérale (La riqueza mineral) de Heron de Villefosse (18101819); la Théorie de la mécanique usuelle, ou, Introduction à l'étude de la mécanique appliquée aux arts (Teoría de la mecánica usual, o, Introducción al estudio de la mecánica aplicada a las artes) de Borgnis (1821), o el Traité de mécanique industrielle (Tratado de mecánica industrial) de M. Christian (1822-1825).

La École Polytechnique se ideó con la pretensión de convertirla en una plataforma educativa que influyese en el desarrollo tecnológico y, por defecto, en el desarrollo económico e industrial de Francia, pues tras la Revolución francesa, que inaugura la era de la razón industrial, la educación se convierte en el motor del progreso de la sociedad. Se creó también, apoyada en una muy sui generis confianza en la jerarquía, en la garantía del orden como principio del progreso, y más aún, en la búsqueda de un consenso social tácito basado en la eficiencia social y económica, concebidas en su sentido más amplio.

Recordemos que la École Polytechnique se constituyó básicamente sobre tres pilares o ejes vertebradores: una ideología específica, la ideología saintsimoniana asociada al industrialismo y al espíritu fáustico del hombre postrevolucionario; una filosofía, el cientismo/cientificismo o positivismo; y un instrumento, las matemáticas y el cálculo, generalizables a todo el ordo material y espiritual del hombre, incluida la sociedad. Si bien tras su primera década de existencia adquiere la impronta napoleónica del Imperio, a pesar de sus principios jacobinos y meritocráticos, como escuela tenía una estructura elitista, de carácter cuartelario y autoritario. De hecho, se puede decir que reflejaría jerárquica y meritocráticamente la sociedad platonizante descrita por el pensamiento politécnico. Sus alumnos eran los hijos de una burguesía (profesionales liberales, funcionarios, banqueros, etcétera) que se estaba consolidando en Francia y que podía permitirse económicamente este tipo 
de educación elitista, pues la formación en la École Polytechnique era muy costosa, aunque la selección de los alumnos se hacía con base en las competencias intelectuales de los mismos mediante arduos exámenes y pruebas de acceso y admisión.

Esta institución de enseñanza superior fue el ámbito más concreto donde se produjo la génesis del pensamiento politécnico, convirtiéndose en un hito capital en la historia de la ingeniería, de la ciencia aplicada, y ciertamente, en la historia de los reformadores y de los ingenieros sociales y del pensamiento sociológico. De hecho, al interior de la École se producían de forma recurrente importantes movimientos políticos e ideológicos, movilizándose, igualmente y de forma espontánea, una ingente actividad intelectual orientada a prácticamente todos los ámbitos y disciplinas del saber, de la ciencia y del conocimiento, tanto de las disciplinas naturales (la Matemática, la Física, la Química, la Biología, entre otras) como de las sociales (la Economía política, la Demografía o Aritmética política, o la nueva ingeniería social, entre otras).

De hecho, en la École Polytechnique se produjeron recurrentes conflictos entre sus miembros y las autoridades, especialmente con respecto a la aplicación de políticas de carácter conservador y reaccionario; hecho que se acusaba aún más por la estrecha vinculación entre el poder y los ámbitos científico y docente. La vocación reformadora de la institución, en los ámbitos social y político, la pone de manifiesto Maurice Daumas en la siguiente cita de su monografía sobre Arago (Daumas, 1987): “L'École n'est pas seulement un centre scientifique, elle attire dans son orbite tous ceux qui rêvent de révolution sociale après la Révolution politique; des hommes comme Clouet, Ferry, Saint-Simon répandent leurs doctrines dans les milieux intellectuels qui gravitent autour de l'École"4 (p. 24).

A partir de la profunda reflexión sobre la ingeniería y la reforma social que es estimulada dentro de la institución y que aprenden sus ingenieros y sociólogos politécnicos, la teoría sobre la sociedad se va abriendo camino hacia la sociología como una disciplina científica plenamente emancipada y eminentemente programática. Si el pensamiento liberal asociado a la economía política pretende la limitación del poder del Estado y del poder político, los ingenieros y sociólogos politécnicos aspiran a su conquista para transformar ordenadamente la sociedad, deshecha tras el colapso posrevolucionario.

Los politécnicos llegaron a constituirse prácticamente en una casta, en un sacerdocio y en un auténtico apostolado de raigambre cuasi masónica; y a lo largo de toda la historia de la institución se ha hablado de un espiritu o un genio politécnicos. De hecho, Auguste Comte, el espíritu más sincrético, iluminista, utópico y representativo del pensamiento social politécnico, manteniendo la ambigua posición de la religión en el ámbito de lo que aquí vamos a referirnos como pensamiento politécnico, según Mises (2001), "quiso reemplazar el cristianismo por una nueva religión e incluso arbitró una mujer que había de ocupar el puesto de la Virgen" (p. 88). Por su parte, si bien el objetivo napoleónico con este centro educativo, creado por la Convención (la asamblea de gobierno de la Revolución), era dotar al ejército de excelentes cuadros y oficiales, y desarrollar la ingeniería militar en todos sus enfoques y sus especialidades, en la École Polytechnique también se desarrolló la ingeniería civil; de hecho, más de la mitad de los alumnos egresados hasta el año 1830 trabajaban en el ámbito de los servicios públicos y en el diseño de las grandes redes de infraestructuras públicas que marcarán el inicio de una época en cuanto a la consideración del papel del Estado en el ámbito del bienestar y en el terreno económico.

En contraposición a la École Normale, que tenía una proyección y una orientación netamente teóricas, la École Polytechnique, ubicada en sus inicios en las dependencias del Palais Bourbon, se consagró a la tecnología 
y a la ciencia aplicada. Estamos hablando, manifiestamente, de tiempos de un saber aplicado; de la materialización, en el ámbito de la acción, de una Idea universal que empieza a despuntar en la Modernidad, especialmente para poder actuar e incidir con éxito sobre la resistente Naturaleza, a la que ya no se contemplaba en el sentido o bajo el prisma aristotélico o clásico (la Naturaleza ya no es un ejemplo a imitar, sino que empieza a ser un recurso, un elemento al que hay que dominar) $\mathrm{y}$, subsecuentemente, sobre la sociedad. No obstante, aunque parezca paradójico, en la institución se mantuvo el privilegio teórico por encima del saber aplicado, siendo, en este sentido, una institución ejemplar y en la que se llegó a establecer un equilibrio feraz, beneficiosamente dialéctico aunque un tanto problemático, entre teoría y saber aplicado (el privilegio de la teoría era el privilegio comteano de la ciencia, como soporte del saber del hombre y de la organización de la sociedad, del industrialismo, en definitiva, de Saint-Simon).

Como he mencionado anteriormente, la École Polytechnique fue fundada en 1794, bajo la inspiración de la Convención y concebida con una perspectiva netamente civil e igualitariojacobina. Posteriormente, en 1804, Napoleón le dio un estatus militar y a partir de ese año y durante una década (1804 a 1814), será un periodo abiertamente marcado por la exaltación de los valores jerárquico-militares que determinarán, de una forma $\mathrm{u}$ otra, la historia posterior de la institución académica durante todo el siglo XIX (Shinn, 1980).

Si bien los antecedentes institucionales de la École Polytechnique se pueden determinar con suficiente exactitud a lo largo del Antiguo Régimen, y en concreto en la segunda mitad del siglo XVIII, los antecedentes culturales de la misma son de naturaleza mucho más difusa y dispersa, aunque obviamente están ligados a la configuración y evolución histórica del ingeniero en las sociedades occidentales posfeudales: haciendo una breve y para nada exhaustiva sinopsis histórica, podemos partir del periodo renacentista, considerado como un tiempo histórico excepcional para el desarrollo de la ingeniería moderna, y a lo largo del cual empieza a consolidarse de forma notoria la profesión del ingeniero humanista que dará lugar, a partir de finales del siglo XVIII y con la gran coartada de la Revolución industrial y productiva que acontecerá en Inglaterra, al ingeniero de Estado y al ingeniero social que caracterizará de forma inequívoca a la École Polytechnique, al pensamiento politécnico, y, concretamente, a los pensamientos tanto del conde de Saint-Simon como de Auguste Comte (el ingeniero filósofo/estadista).

En efecto, durante el Renacimiento la profesión y la figura del ingeniero adquieren una especial relevancia en el imaginario $\mathrm{y}$ en el inconsciente colectivo, que tienen como referencia ingeniero humanista del renacimiento (Gourisse, 1999). Hablamos de un ingeniero de carácter multidisciplinar, propio de la caracterización antropológica del quinientos, humanista, sin formación específica y conocedor de todas las técnicas en los distintos ámbitos de la ingeniería; un hombre que domina la ciencia, la técnica, $y$, por supuesto, es un artista polivalente que opera con multitud de materiales, diseños y formatos acordes con el desarrollo y las demandas públicas y privadas propias de la época. Aún más, Daniel Gourisse (1999) señala: “il n'est pas homme de corporations. Il forme avec les autres ingénieurs une communauté d'esprit internationale. Savant humaniste universel, il ne connaît pas de frontières et parcourt l'Europe" ${ }^{\prime 5}$ (p. 1).

Durante la época del Renacimiento es difícil distinguir al ingeniero del arquitecto o del artista de las bellas artes. Según hace saber Larry Shiner (2004), es la creación de la École des Ponts et Chauseés el hito histórico que viene a marcar definitivamente el

[...] comienzo de la separación entre arquitectos e ingenieros, separación que se acentuaría 
más tarde con el establecimiento de la École Polytechnique en 1794. No obstante, ambas, la École Polytechnique y la École des Beaux Arts (sucesora de las academias de pintura, escultura y arquitectura después de la Revolución) ofrecían cursos de arquitectura, hecho que refleja el carácter "mixto" de ésta (p. 157).

Con la conformación histórico-cultural del ingeniero humanista del Renacimiento, el futuro de las grandes escuelas de ingenieros civiles (Ponts et Chaussées, Mines, Polytechnique...) podría estar en cierto modo pronosticado, si bien faltarían todavía unos doscientos años para su materialización efectiva aprovechando el proceso de diferenciación y especialización científica; los años justamente necesarios para que se produjera la gran Revolución científica del Seiscientos, con Newton como máximo representante, la Ilustración francesa del Ochocientos, y, por supuesto, la Revolución industrial inglesa, observada minuciosamente y con admiración por los ingenieros y científicos politécnicos del Continente.

Precisamente, en este periodo posrenacentista y prepolitécnico se desarrollarán dos modelos de ingeniero acusadamente definidos a partir de la mitad del siglo XVIII: por un lado un modelo netamente inglés, y por otro un modelo de carácter continental (Gourisse, 1999). El perfil de ingeniero inglés, sin formación académica especializada y siendo el artífice inequívoco de la primera Revolución industrial en Inglaterra, se apoyará técnicamente en una experiencia y en unas competencias prácticas que pertenecerán propiamente a la esfera de las primeras industrias y de las primeras empresas industriales, los talleres artesanos y los oficios convencionales. Por su parte en el continente europeo y particularmente en Francia, como refiere Daniel Gourisse (1999), la palabra ingeniero tendrá otros matices: concretamente se asocia al ingeniero de Estado, un profesional con un gran sentido corporativo y vinculado a las necesidades de los Estados modernos: protección de las fronteras, construcción de puertos y carreteras, etcétera.
Este segundo modelo, el continental, que no deja de ser un patrón o un tipo ideal en el sentido weberiano del término, estará vinculado a una concepción ideológica positiva de la profesión de ingeniero, cuya formación requiere conocimientos superiores y mucho más avanzados y complejos que los propios o los que se generan a partir de los oficios, o de las experiencias en los talleres artesanos, o en las primeras empresas industriales; conocimientos específicos de matemáticas, física, química, geometría (Monge), etcétera, basados en una sólida y enriquecedora alianza entre la Ciencia y las Artes (la técnica). Sólo a partir de estos conocimientos se podría acometer la revolución social ilustrada desde la ingeniería que planteaba el ingeniero fáustico politécnico.

En términos más concretos y desde un enfoque institucional, entre los antecedentes de la École Polytechnique y de la conformación contemporánea del ingeniero, podemos mencionar a Jean-Baptiste Colbert, ministro de Luis XIV, que fundó en 1675 la primera escuela formal de ingeniería: el Corps $d u$ Génie. Posteriormente, en 1771, en Inglaterra, John Smeaton, el primer ingeniero civil del país, junto con un pequeño grupo de ingenieros, funda la Society of Civil Engineers. Además, surgen dos escuelas superiores de ingeniería antes de la Revolución: en 1747, la École des Ponts et Chaussées; y, en 1783, la École Royale des mines. Específicamente, el embrión institucional y organizacional es la École des Ponts et Chaussée: precursora prerrevolucionaria e inmediata de la gran escuela posrevolucionaria de Francia, la École Polytechnique, heredera de las viejas escuelas y cuerpos de ingenieros del Antiguo Régimen.

En efecto, así fue. El corps des Ingénieurs des Ponts et Chaussées (constituido básicamente por funcionarios, burócratas y tecnócratas que se ocupaban del interés general), fue creado en 1716 bajo el modelo del Corps du Génie Militaire, responsable de las fortificaciones militares, y cuyo objetivo era contribuir a la 
construcción de las grandes infraestructuras públicas del Estado. Al respecto, Antoine Picon (1994) señala que la profesión de ingeniero en Francia estaba encuadrada en el ámbito del Estado, al contrario de lo que ocurriría en Inglaterra (p.77-99). Como evolución endógena del Corps, en 1747 se crea la École des Ponts et Chaussées, la primera gran escuela de ingenieros de Francia, con el objetivo de convertirla en un gran centro docente para la formación específica de los ingenieros de Estado: formar alumnos en ingeniería civil, construcción, transportes, etcétera. El primer director de esta escuela, cargo que ocupó desde la creación de la institución hasta su muerte (1747-1794), fue el ingeniero y erudito, y que participó activamente en la Enciclopedia de Diderot y D’Alembert, Jean-Rodolphe Perronet. La École empezó sin profesores y entre los primeros alumnos, autodidactas ellos, se encontraban los conocidos Philippe Lebon, Bernardin de SaintPierre, Méchain y Brémontier.

Los ingenieros y la École des Ponts et Chaussées recibieron importantes privilegios y sus actividades tenían características de monopolio, por lo que tras la gran Revolución, esta institución encuentra numerosos detractores. De ahí que se opte por crear, a través de una ley de 28 de septiembre de 1794, la gran École posrevolucionaria, la École Centrale des Travaux Public, que un año más tarde tomará el nombre definitivo de École Polytechnique, y que agrupa en una sola, las escuelas Ponts, Mines et Génie. Se mantiene la École des Ponts et Chaussées, muy desestructurada y desorganizada tras la muerte, en 1794, de Perronet, al que sucederá Lamblardie, pero con un carácter de escuela aplicada. La École Polytechnique será la genuina y gran escuela posrevolucionaria, símbolo inequívoco y emblemático de la construcción del nuevo sistema educativo napoleónico, y que, como ya hemos advertido, en 1804 adopta un orden militar, una bandera y un célebre lema de factura fáustica: "Pour la Patrie, les Sciences et la Gloire". De esta manera, la École des Ponts et Chaussées será concebida para formar técnicos y cuadros cualificados en el ámbito de la construcción y diseño de caminos, carreteras, canales, puentes, trazados ferroviarios, etcétera; y la École Polytechnique posrevolucionaria, se concebirá para combatir la escasez de cuadros civiles y militares.

Ya en la École des Ponts et Chaussées es latente la sólida creencia de la importancia del ingeniero en la labor de eliminar las barreras sociales a través de la supresión de las barreras físicas y geográficas, integrando al país y a la sociedad en una unidad física y espiritual. El ingeniero, cuestión que será fundamental en la École Polytechnique y en la creación de la sociología, será una agente activo imprescindible para la vertebración de la sociedad, permitiendo una mayor velocidad en los flujos del protocapitalismo contemporáneo a través de la creación de las grandes redes e infraestructuras viarias, ferroviarias, hidráulicas, etcétera. En todo ingeniero francés de los siglos XVIII y XIX hay siempre un reformador social o un utopista, de ahí que sea en el seno del pensamiento politécnico donde nazca la sociología como disciplina o ciencia social. Como dice Picon (1994), este hecho pone de manifiesto la influencia fisiocrática en las escuelas de ingenieros a través de su lema "laisser faire-laisser passer" (pp. 77-99). Como prueba de la importante labor en la creación de las grandes redes camineras, Picon estima, mediante unas estadísticas contundentes cuya fuente es Perronet (1963), que a la altura del año 1776 se había construido una infraestructura caminera de unos 13.932 kilómetros de longitud.

Continuando con la historia de la École Polytechnique, hay que destacar que el gran artífice de este prestigioso y novedoso establecimiento, que encajará plenamente en el credo napoleónico, fue Gaspard Monge, el padre de la geometría descriptiva y, junto con Euler, de la geometría diferencial; su objetivo era asegurar la superioridad de la reciente República en el ámbito del saber científico y técnico. En efecto, este íntimo amigo de 
Napoleón fue el fundador y director en dos ocasiones de la École Polytechnique, antes de su militarización en 1804. En el proyecto colaboró Lazare Carnot, y fue especialmente apoyado por Prieur de la Côte d'Or, miembro del Comité de Salut public durante la Terreur. La institución contó con docentes o con estudiantes que eran, o más tarde serían, los intelectuales, científicos, sabios, tecnólogos e ingenieros más relevantes de la época, así como de la historia de la ciencia moderna: Berthelot (junto Berthelot con Lavoisier instauraron la química moderna), Lamblardie, Chaptal, Guyton de Morveau, Laplace, Coriolis, Lamé, Fourier, Hassenfratz, Vauquelin, Poinsot, SylvestreFrançois Lacroix, Jean-Victor Poncelet, Poisson, Liouville, André-Marie Ampère, GayLussac, Louis Jacques Thénard, François Arago, Cauchy, Legendre, Chasles, Sturm, Malus, Dulong, Volta, Fresnel, Dupuit, Biot, Rumford, el célebre Alexander von Humboldt, Le Play, entre otros. Como gran novedad de esta escuela posrevolucionaria frente a la École des Ponts y Chaussées y su competidora, la École royale du Génie de Mézières, habría que mencionar, además, la importancia que tuvieron ciencias como la matemática, la física y la química, así como una influencia clara y patente de la Ilustración, transmitida mediante una confianza desmedida en las ciencias, en el saber teórico y especialmente, en el saber lexicográfico de la Enciclopedia.

Esta concentración de los principales científicos del mundo consolidaba a Francia como el primer país en promocionar la ciencia y el saber práctico, hecho que empezaba a ser latente en la segunda mitad del siglo XVIII junto con la Ilustración y el proceso editorial de la Enciclopedia, y que posteriormente se consolidaría con la creación de la École Polytechnique. La hegemonía francesa se traducirá en hitos tan importantes como la racionalización y maduración de una multitud de disciplinas científicas que aún estaban en una etapa cuasi-mitológica o metafisica, "conjetural", dirá Burdin: Lagrange racionalizará la Mecánica, Lavoisier la
Química, Laplace la Astronomía (la mecánica celeste), Buffon hará lo propio con la Biología, etcétera. Favorecida por el traslado de la actividad científica desde la Académie Royale des Sciences de París a la École Polytechnique, a la École Normale y al Institut de France, la ciencia alcanzaría un grado inusual de profesionalización (Castrillo, 1985). Sin precedentes en la historia, tanto desde el punto de vista cuantitativo como cualitativo, así en Francia como en todo el mundo, se desarrolló una comunidad científica con una dimensión que estimuló la competencia y la rivalidad entre los propios hombres de ciencia, apoyando en consecuencia la producción cientifica y desarrollando un entorno propicio para la innovación, la creatividad y la ordenación (prácticamente la que se vive en la actualidad) de los saberes y disciplinas de la ciencia. Esta comunidad científica, sin lugar a dudas, superaba las fronteras políticas; era una red supranacional de conocimiento y de ciencia que establecería de forma inequívoca las bases de la comunidad científica de nuestros días.

Estos prestigios científicos, ingenieros y sociólogos politécnicos conceptualizaron la definición de sociedad como una genuina exaltación y radicalización de la idea Ilustrada y Fisiocrática, es decir, de la literalidad de la razón, de sus extralímites y del espíritu enciclopédico, de la unidad y del concepto holístico y consiliente del saber. Prácticamente los científicos, ingenieros y sociólogos de la École Polytechnique se hicieron eco de una suerte de determinismo ecuacional que soportaba todo el entramado material y natural del mundo conocido y del mundo remoto: la naturaleza se podía ajustar a ecuaciones precisas y relativamente sencillas; de ahí a la formulación de leyes generales que rigen horizontalmente para la sociedad, para la política y para la economía, sólo habría un paso.

A finales del siglo XVIII la ingeniería deja de ser un mero oficio y pasa a ser una auténtica profesión en su sentido más evolucionado, especialmente relevante en un ambiente de euforia 
y de optimismo antropológico sin precedentes, motivado por el espíritu fáustico spengleriano que define al hombre contemporáneo que surge tras los grandes procesos revolucionarios. La ingeniería será apoyada por el Imperio, y la École Polytechnique se convertirá en la institución de enseñanza tecnológica por excelencia y origen manifiesto de las escuelas politécnicas actuales. Para el físico Biot (1803), la École Polytechnique tenía un triple objetivo: "former des ingénieurs pour les différents services, répandre dans la société des hommes éclairés et exciter les talents qui pourraient avancer les sciences. Rien ne fut négligé pour cette importante destination" (Allain, 1969, p. 203).

La euforia en la institución docente postrevolucionaria caló muy profundamente. La mente y el conocimiento del hombre poscartesiano no tenían límites; la emancipación definitiva del mismo, mediante la autoconciencia y la ampliación de los límites metafísicos del espíritu y de la razón, se presumía inminente bajo las directrices del progreso, y, en concreto, del progreso tecnológico, el culto a la ciencia, la artificialidad, el constructivismo ilimitado (de grandes infraestructuras físicas, pero también de grandes constructos sociales), la educación práctica y pragmática, y el positivismo lagrangiano. De la institución emanaba un claro espíritu sintético, práctico, fáustico/mefistofélico, pragmático, determinista, unificador, universalista, consiliente y esencialmente fisicalista, capaz de concebir las leyes generales que soportan y regulan el mundo natural y social en constante intercambio.

Sin duda, los científicos, ingenieros y sociólogos politécnicos se creyeron capaces de concretar y materializar una suerte de Idea hegeliana que sobrevolaba Europa desde los inicios de la Modernidad y que arraigaba y estaba latente en el Renacimiento maquiavélico y posteriormente en la Revolución científica y, más tarde, en el pensamiento ilustrado. La visualización general y trascendental de esta Idea es la que aceleró el proceso de radicalización de los principios ilustrados. Se produce la gran Revolución política y económico-productiva (industrial) que inaugura la historia contemporánea, así como el nacimiento de la democracia moderna (es la definitiva toma de conciencia de la sociedad), marco necesario para la conformación de la sociología como disciplina científica, y la inequívoca revelación del mercado (en sentido moderno) como elemento y principio vertebrador de la actividad económica. Mucho antes que un relevo sistemático y recurrente del poder político, se presenta como una realidad de carácter espiritual y gnoseológico, una realidad relacionada con la autoconciencia y la materialización del pensamiento colectivo (estamos asistiendo, sin lugar a dudas, a los principios de la creación de la opinión pública).

Se puede decir que el pensamiento que florece en la École Polytechnique, al menos en las cuatro décadas siguientes a su creación, era una radicalización del pensamiento ilustrado y enciclopédico (una segunda Ilustración, esta vez fáustica y extrema: creación de dictaduras pedagógicas, sociedades jerarquizadas con base en la lógica de la producción [la sociedadfábrica], equiparación de la ciencia a una experiencia de índole religiosa, casi con el estatuto de una vedette, etcétera) trasladado inmediatamente a la reorganización social a través de la capacidad de programación de los ingenieros/sociólogos politécnicos y del ansia extrema, de índole protohegeliana, de materialización de la Idea y del Espíritu en el orden de las cosas, superando la dualidad cartesiana de la Modernidad. Se asentarán definitivamente los principios corporativistas produciendo un escenario ideal para su transmisión a muchos órdenes de la vida: el militar, el científico o el burocrático.

El ingeniero social ponía como evidencia el principio socrático-platónico de unidad de la ciencia y de la política, de la praxis humana en definitiva. Además, la radicalización del pensamiento enciclopédico era animada por el optimismo productivista y ascendente de la 
Revolución industrial, con su alumbramiento axial de la división del trabajo, y su vinculación con la idea de progreso, un progreso racional, mecánico, tecnológico, en muchos casos tautológico, etcétera. No obstante, esta utopía se irá diluyendo conforme nos alejamos paulatinamente en el tiempo de la gran Revolución de 1789, produciéndose, como advierte Picon, tensiones importantes entre la teoría y el saber práctico o aplicado, entre la ciencia de Gaspard Monge y la tradición ilustrada de Lagrange y la práctica ingenieril; es decir, entre la École Polytechnique y la École des Ponts et Chaussées, así como dentro de la propia École Polytechnique.

\section{Conclusiones}

De este establecimiento, una institución netamente republicana, nace un proyecto de gobierno en el que la ciencia y la industria son sus soportes fundamentales. Y los dos, según Saint-Simon -y sus seguidores en la institución y fuera de ella-, estarán unidos por un profundo enlace devoto que volvería a "restaurar la unidad de las ideas religiosas rota desde la Reforma" (Engels, 1968, p. 41), base del consenso y de la nueva paz social que definen los sociólogos politécnicos. Como advierte Engels (1968), en Saint-Simon se da el embrión de la total absorción de la política por la economía. La ciencia y los organizadores sociales, los fisiólogos sociales, provocarán el descrédito de los filósofos, moralistas y metafísicos. Por la ciencia, la educación y el desarrollo tecnológico se alcanzaría el gran consenso de la humanidad: positivismo e industria. Además, la enseñanza para Saint-Simon, como para otro proeuropeísta e importante y célebre pedagogo, Juan Amós Comenio (Truyol \& Serra, 1975), teniendo aquél como modelo la École Polytechnique, será fundamental para la consecución de la paz perpetua que rescata de la ilustración kantiana, para la aparición de las lealtades superiores (una Europa unida con rasgos novalisianos), e, igualmente, para la consecución del consenso necesario para la realización material de la utopía industrial y positiva que planteaban los sociólogos politécnicos. La idealización de una Europa supranacional y desteleologizada de Saint-Simon, arraigada en un sólido, estable y profundo acuerdo anglo-francés, sería la mayor realización política producida por el espíritu ilustrado.

Este ambiente espiritual, de acusado carácter fáustico, propiciará la concepción sofocrática del gobierno de la sociedad a través de la ciencia; en concreto, a través de una disciplina eminentemente platónica: la sociología; una ciencia, que en el sentido propuesto por Ernest Renan, pretendería organizar bajo sus principios a la humanidad a través de la consecución de una sociedad hiperracional, resuelta al modo geométrico spinoziano $\mathrm{y}$, obviamente, basada en los principios de la naturaleza y en sus principales dictados, caracterizados esencialmente por el principio de necesidad.

\section{Notas}

1 "Los temas de enseñanza, sus ilustres profesores, los distinguidos alumnos que ya ha proporcionado a los diversos servicios públicos, bastarían para defenderlo".

2 "La École Polytechnique, organizada en el año III, tuvo como profesores a los más ilustres eruditos, Lagrange, Prony, Monge, Berthollet, Fourcroy, Chaptal, Vaquelin y Guyton de Morveau. Fue entendida para entrenar ingenieros civiles y militares, para enseñar los principios generales de la ciencia."

3 El mito de Fausto hace referencia a la leyenda del hombre que vendió su alma al diablo a fin de lograr el conocimiento y los placeres mundanos.

4 "La École no es sólo un centro científico, sino que atrae a su órbita a todos los que sueñan con la revolución social después de la Revolución Política; hombres como Clouet, 
Ferry y Saint-Simon difundieron sus doctrinas en los círculos intelectuales que giraban alrededor de la École.”

5 "No es un hombre de corporaciones. Con los otros ingenieros, formó una comunidad de espíritu internacional. Un humanista universitario universal, no conoce fronteras y viaja por Europa".

6 "Formar a los ingenieros para los diferentes servicios, para difundir a los hombres ilustrados en la sociedad, y para estimular los talentos que podrían hacer avanzar a las ciencias. Nada fue descuidado para este importante destino".

\section{Referencias}

Allain, E. (1969). L'oeuvre scolaire de la Révolution 1789-1802. Nueva York: Burt Franklin.

Álvarez-Uría, F., \& Varela, J. (2004). Sociología, capitalismo y democracia. Madrid: Ediciones Morata.

Belidor, N. (1819). Architecture hydraulique. París: Firmin Didot.

Biot, J. B. (1803). Essai sur l'histoire génerale des sciences pendant la révolution française. París.

Borgnis, M. J. A. (1821). Théorie de la mécanique usuelle, ou, Introduction à l'étude de la mécanique appliquée aux arts. París: Bahelier, Libraire.

Cardwell, D. (2001). Historia de la tecnología. Madrid: Alianza.

Castrillo, P. (1985). Ensayo filosófico sobre las probabilidades. Madrid: Alianza.

Christian, M. (1822-1825). Traité de mécanique industrielle. París: Bachelier, Libraire.
Coller, X. (2003). Canon sociológico. Madrid: Tecnos.

Daumas, M. (1987). Arago. La jeunesse de la science (Dir. Jean Dhombres). Paris: Belin.

Engels, F. (1968). Del socialismo utópico al socialismo científico. Madrid: Ricardo Aguilera Editor.

Fourcy, A. (1828). Histoire de l'École Polytechnique. París: Imprimerie de A. Belin.

Gourisse, D. (1999). Histoire et avenir des ingénieurs en Europe. Académie des Sciences morales et politiques. Recuperado de https:/www.asmp.fr/travaux/communications/1999/gourisse.pdf.

Guenyveau, A. (1810). Essai sur la science des machines. Lyon: De l'Imprimérie de J. B. Kindelem.

Hachette, M. (1811). Traité élémentaire des Machines. París: Coret, Libraire Editeur.

Hayek, F. A. (2003). La contrarrevolución de la ciencia. Madrid: Unión Editorial.

Heilbron, J. (1995). The Rise of Social Theory. Minneapolis: University of Minnesota Press.

Heron de Villefoss, A. M. (1810-1819). De la richesse minérale. París.

Langins, J. (1987). La République avait besoin de savants. Les débuts de l'École polytechnique: l'École centrale des travaux publics et les cours révolutionnaires de l'an III. Paris: Belin.

Lanz, J. M., \& Betancourt, A. (1808). Essai sur la composition des machines. París: Imprimerie impériale. 
Mises, L. V. (2001). La acción humana. Tratado de Economía. Madrid: Unión Editorial.

Negro, D. (1987). Comte: Positivismo y Revolución. Madrid: Cincel.

Perronet, J.-R.(1963). Etat des plans des grandes routes et chemins du royaume. París: École Nationale des Ponts et Chausséss.

Picavet, F. J. (1975). Les idéologues. Nueva York: Arno Press.

Picon, A. (1994). “Die Ingenieure des Corps des Ponts et Chaussées Von der Eroberung des nationalen Raumes zur Raumordnung ”. En A. Grelon \& H. Stück (Dirs.), Ingenieure in Frankreich, 1747-1990 (pp. 77-99). Nueva York: Francfort.
Prony, R (1790, 1796). Nouvelle architecture hydraulique. París: De l'Imprimerie de Didot Fils Ainé.

Shiner, L. (2004). La invención del arte. Una historia cultural. Barcelona: Ediciones Paidós Ibérica.

Shinn, T. (1980). L'École Polytechnique, 17941914. Savoir politique et pouvoir social. Paris: Presses de la Fondation Nationale des Sciences Politiques.

Truyol, I., \& Serra, A. (1975). De la reorganización de la sociedad europea. Madrid: Instituto de Estudios Políticos. 
